\title{
Convexity invariance of voxel objects under rigid motions
}

\author{
Phuc Ngo \\ Université de Lorraine \\ LORIA, UMR 7503, France \\ hoai-diem-phuc.ngo@loria.fr
}

\author{
Nicolas Passat \\ Université de Reims \\ Champagne-Ardenne \\ CReSTIC, France
}

nicolas.passat@univ-reims.fr

\author{
Yukiko Kenmochi \\ Université Paris-Est \\ LIGM, CNRS, France \\ Isabelle Debled-Rennesson \\ Université de Lorraine \\ LORIA, UMR 7503, France \\ yukiko.kenmochi@esiee.fr isabelle.debled-rennesson@loria.fr
}

\begin{abstract}
Volume data can be represented by voxels. In many applications of computer graphics (e.g. animation, simulation) and image processing (e.g. shape registration), such voxel data require manipulations. Among the simplest manipulations, we are interested in rigid motions, namely motions that do not change the shape of voxel objects but do change their position and orientation. Such motions are well-known as isometric transformations in continuous spaces. However, when they are applied on voxel data, some important properties of geometry and topology are generally lost. In this article, we discuss this issue, and we provide a method for rigid motions of voxel objects that preserves the global convexity properties of objects, with digital topology guarantees. This method is based on the standard notion of H-convexity, and a new notion of quasi-regularity.
\end{abstract}

\section{INTRODUCTION}

Representation of volume data is an important element in 3D imaging applications. One of the commonly used representations relies on digital models, namely sets of voxels (3D pixels), as it provides a uniform and simple framework, that allows for describing easily the topology and geometry of objects. Voxel data are defined on the 3D Cartesian grid $\mathbb{Z}^{3}$. Each voxel - unit cube centered on a grid point - is represented by its integer coordinates in $\mathbb{Z}^{3}$.

Voxel data are frequently involved in computer graphics (e.g. animation in games, simulations) and image processing techniques (e.g. object tracking, shape registration), and in applications related to 3D images (e.g. medical imaging). In such applications, voxel data are often required to be manipulated in $\mathbb{Z}^{3}$. Among simple operations, we are interested in rigid motions which refer to changing the position and orientation of a 3D object without deforming its shape. In this article, we consider rigid motions composed by rotations and translations.

Applying rigid motions on $\mathbb{Z}^{3}$ is generally assumed to be simple and to preserve object shapes (geometry and topology) due to their isometry properties on $\mathbb{R}^{3}$. However, such invariance properties are no longer satisfied with voxel objects. Rigid motions on $\mathbb{Z}^{3}$, called digital rigid motions, are generally performed in the associated continuous space and followed by a sampling operation (also called digitization) of the result. Because of this digitization step (and also of numerical approximations of the continuous transformations), digital rigid motions do not satisfy connectivity and geometry preservation, as exemplified in Fig. 1. It should be noticed

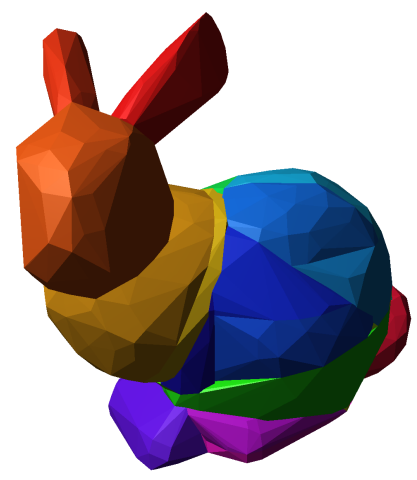

(a)

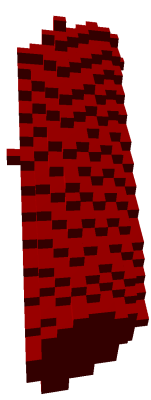

(c)

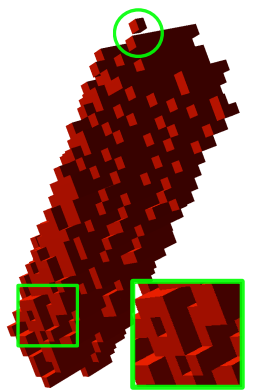

(d)

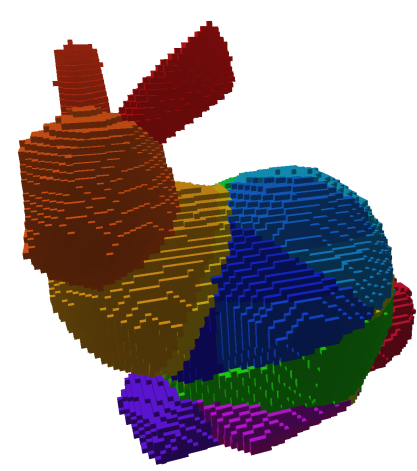

(b)

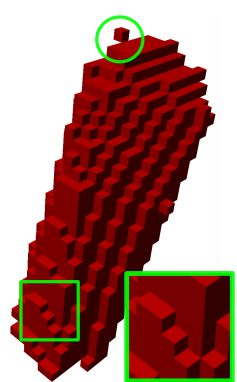

(e)

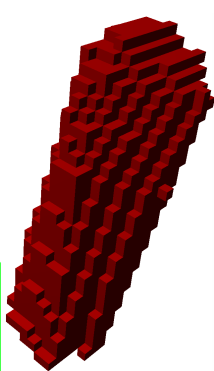

(f)
Fig. 1. (a) Convex decomposition of Bunny mesh. (b) Voxel representation of (a). (c) A convex part — right ear - of (b). (d) Rigid motion of (c) followed by digitization where convexity and connectivity are lost, see green areas. (e) Rigid motion of (c) based on half-plane representation where convexity is preserved, but not the connectivity, see green areas. (f) Our proposed method; both convexity and connectivity are preserved.

that, contrary to $\mathbb{R}^{3}$, convexity does not imply connectivity on $\mathbb{Z}^{3}$ (see Fig. 1(e)). Several issues of digital rigid motions have been identified and discussed in [1]-[3]. In a context of image processing and analysis, rigid motion followed by digitization (or interpolation) is generally used for transforming voxel objects (see Sec. II). However, this can generate artifacts and lead to undesired biases of connectivity and geometry in the final result (see Fig. 1(d)).

In this article, we propose an alternative method devoted to qualitatively preserving convexity properties of voxels objects under rigid motions. More precisely, the proposed method 
is based on a representation of the objects by (digital) halfspaces. Based on this representation, we establish a property of the convex hull of voxel objects that allows for the preservation of convexity and connectivity under rigid motions.

\section{PROBLEM OF RIGID MOTIONS IN $\mathbb{Z}^{3}$}

\section{A. Objects and rigid motions in $\mathbb{R}^{3}$}

Let us first consider an object $X \subset \mathbb{R}^{n}$ in the Euclidean space $\mathbb{R}^{3}$ as a closed, connected, bounded subset of $\mathbb{R}^{3}$. Rigid motions on $\mathbb{R}^{3}$ are defined by:

$$
\begin{array}{lll}
\mathfrak{T}: & \mathbb{R}^{3} & \rightarrow \mathbb{R}^{3} \\
& \mathrm{x} & \mapsto R \mathrm{x}+\mathrm{t}
\end{array}
$$

where $R$ is a rotation matrix and $\mathrm{t} \in \mathbb{R}^{3}$ is a translation vector. Such transformation $\mathfrak{T}$ is bijective and isometric. In particular, $\mathfrak{T}(\mathrm{X})$ has the same geometry and connectivity as X.

\section{B. Digitization of objects and connectivity}

The digitization of $\mathrm{X}$ is defined by the intersection of $\mathrm{X}$ and $\mathbb{Z}^{n}$, called Gauss digitization [4]. Such digitization operator is intuitive. However, it may cause shape alterations. In this context, conditions for guaranteeing connectivity of digitized objects have been studied for $n=3$ [5], [6] based on a notion of regularity, originally proposed for $n=2$ [7], [8].

Definition 1 ( $r$-regularity): An object $\mathrm{X} \subset \mathbb{R}^{3}$ is $r$-regular if for each boundary point of $\mathrm{X}$, there exist two tangent open balls of radius $r$, lying entirely in $\mathrm{X}$ and its complement $\overline{\mathrm{X}}$, respectively.

This notion is based on classical concepts of differential geometry. It establishes a topological link between a continuous shape and its digital counterpart, based on the notion of connectivity [6]. Given a point $p \in \mathbb{Z}^{3}$, the neighborhood of $p$ is defined by $\mathcal{N}(p)=\left\{q \in \mathbb{Z}^{3}:\|p-q\|_{1} \leq 1\right\}$. We say that a point $q$ is adjacent to $p$ if $q \in \mathcal{N}(p) \backslash\{p\}$. From the reflexivetransitive closure of this adjacency relation on a finite subset $X \subset \mathbb{Z}^{3}$, we derive the connectivity relation on $X$, which is an equivalence relation. If there is exactly one equivalence class for this relation, then we say that $\mathrm{X}$ is connected.

Proposition 1 (From [6]): If a (connected) object $\mathrm{X} \subset \mathbb{R}^{3}$ is $r$-regular with $r \geq \sqrt{3} / 2$, then the digitized object $\mathrm{X} \cap \mathbb{Z}^{3}$ is connected.

\section{Digital objects and rigid motions in $\mathbb{Z}^{3}$}

A rigid motion $\mathfrak{T}$, when applied on a $3 \mathrm{D}$ digital object $X \subset \mathbb{Z}^{3}$, generally leads to $\mathfrak{T}(X) \not \subset \mathbb{Z}^{3}$. Therefore, in order to get it back into $\mathbb{Z}^{3}$, we need a digitization operator $\mathfrak{D}: \mathbb{R}^{3} \rightarrow \mathbb{Z}^{3}$. Such operator is generally defined as the standard rounding function. The discrete analogue of $\mathfrak{T}$ is then defined as $\mathcal{T}_{\text {point }}=\mathfrak{D} \circ \mathfrak{T}_{\mid \mathbb{Z}^{3}}$. We note $\mathcal{T}_{\text {point }}(\mathrm{X})=$ $\left\{\mathcal{T}_{\text {point }}(\mathrm{p}) \mid \mathrm{p} \in \mathrm{X}\right\}$.

Figure 1(d) shows that the geometry and topology are generally not preserved by $\mathcal{T}_{\text {point }}$. Motivated by this issue, we focus on the fundamental properties of convexity and connectivity. We present a method for rigid motions on $\mathbb{Z}^{3}$ that preserves these properties.

\section{Digital CONVEXITY}

An object $\mathrm{X} \subset \mathbb{R}^{n}$ is said to be convex if for any pair of points $\mathrm{x}, \mathrm{y} \in \mathrm{X}$, every point on the straight line segment joining $\mathrm{x}$ and $\mathrm{y}$, defined by $[\mathrm{x}, \mathrm{y}]=\{\lambda \mathrm{x}+(1-\lambda) \mathrm{y} \mid 0 \leq$ $\lambda \leq 1\}$, is also within $\mathrm{X}$. This continuous notion, however, cannot be directly applied to digital objects $X$ in $\mathbb{Z}^{n}$. Indeed, for $\mathrm{p}, \mathrm{q} \in \mathrm{X}$ we have $[\mathrm{p}, \mathrm{q}] \not \subset \mathbb{Z}^{n}$ as soon as $\mathrm{p} \neq \mathrm{q}$. In order to tackle this issue, various extensions of the notion of convexity have been proposed in $\mathbb{Z}^{n}$, in particular for $n=2$ [9]-[11]. Among these variations, we adopt the H-convexity notion, initially defined in 2D [9]. H-convexity allows us to develop a method, based on the half-space representation of convex polyhedra, for convexity-preserving rigid motions of $3 \mathrm{D}$ digital objects. The definition of $\mathrm{H}$-convexity requires the notion of convex hull, which is defined for a digital object $\mathrm{X} \subset \mathbb{Z}^{3}$ as:

$$
\begin{array}{r}
\operatorname{Conv}(\mathrm{X})=\left\{\mathrm{x} \in \mathbb{R}^{3} \mid \mathrm{x}=\sum_{\mathrm{p} \in \mathrm{X}} \lambda_{\mathrm{p}} \mathrm{p} \wedge \sum_{\mathrm{p} \in \mathrm{X}} \lambda_{\mathrm{p}}=1\right. \\
\left.\wedge \forall \mathrm{p} \in \mathrm{X}, \lambda_{\mathrm{p}} \geq 0\right\} .
\end{array}
$$

Definition 2 (H-convexity): A digital object $\mathrm{X} \subset \mathbb{Z}^{3}$ is $\mathrm{H}$ convex if $\mathrm{X}=\operatorname{Conv}(\mathrm{X}) \cap \mathbb{Z}^{3}$.

Let us remark that H-convexity does not imply connectivity in $\mathbb{Z}^{3}$ (resp. $\mathbb{Z}^{n}$ ) by contrast to $\mathbb{R}^{3}$ (resp. $\mathbb{R}^{n}$ ). This motivates our choice of assuming that the objects we handle are also connected on $\mathbb{Z}^{3}$. This additional connectivity property of a digital object is crucial for further guaranteeing that $\mathrm{H}$ convexity preservation during rigid motions will allow us to obtain a relevant shape of the digital object.

\section{CONVEXITY AND RIGID MOTIONS ON $\mathbb{Z}^{3}$}

In this section, we propose a method that allows us to preserve the $\mathrm{H}$-convexity of digital objects $\mathrm{X}$ under any rigid motion. As stated in Sec. II, it is geometrically irrelevant to apply directly a digitized rigid motion $\mathcal{T}_{\text {point }}$ on $\mathrm{X}$. Indeed, this may lead to alterations of both connectivity and convexity. To overcome this issue, we first introduce the half-space representation of a H-convex digital object. Then, a (continuous) rigid motion is performed on the set of half-spaces constituting the object. This process is then followed by a redigitization (back to $\mathbb{Z}^{3}$ ) of the intersecting region of the transformed half-spaces, to obtain the transformed $\mathrm{H}$-convex digital object. Furthermore, a condition, namely quasi-r-regularity, on $\mathrm{X}$ is proposed for guaranteeing the connectivity preservation of the transformed objects under a Gauss digitization process.

\section{A. Digital half-space representation}

The representation of convex polyedra with half-spaces called, H-representation - was introduced in [12]. The $\mathrm{H}$ representation is used here to model the H-convex digital objects. Let us consider a $\mathrm{H}$-convex digital object $\mathrm{X} \subset \mathbb{Z}^{3}$, containing at least four non-coplanar grid points of $\mathbb{Z}^{3}$, so that $\mathcal{C}$ onv $(\mathrm{X})$ has a non-null volume. By construction, the coefficients of the half-spaces modeling the polyhedron $\operatorname{Conv}(\mathrm{X})$ are integers; we call these half-spaces integer half-spaces. 
They are computed from the convex hull $\operatorname{Conv}(\mathrm{X})$ of $\mathrm{X}$. In particular, the vertices of $\operatorname{Conv}(\mathrm{X})$ are grid points of $\mathrm{X}$.

$$
\operatorname{Conv}(\mathrm{X})=\bigcap_{\mathrm{H} \in \mathcal{R}(\mathcal{C} \operatorname{Con} v(\mathrm{X}))} \mathrm{H},
$$

where $\mathcal{R}(P)$ is the minimal set of closed half-spaces that constitute the convex polyhedron $P$. Each $\mathrm{H}$ is a closed halfspace as follows:

$$
\mathrm{H}=\left\{x \in \mathbb{R}^{3} \mid \mathrm{n} \cdot x+d \leq 0\right\},
$$

where $\mathrm{n}=(a, b, c) \in \mathbb{Z}^{3}$ with $\operatorname{gcd}(a, b, c)=1$ is the normal vector of $\mathrm{H}$, and $d \in \mathbb{Z}$.

The integer coefficients of $\mathrm{H}$ are uniquely obtained from three incident vertices of $\mathcal{C}$ onv $(\mathrm{X})$ (i.e., forming a triangle on the surface of the convex hull), denoted by $u, v, w \in \mathbb{Z}^{3}$ in clockwise ordered, such that

$$
\begin{aligned}
\mathrm{n} & =\mathrm{n}_{1} \times \mathrm{n}_{2} \\
\mathrm{~d} & =-\mathrm{n} \cdot \mathrm{u}
\end{aligned}
$$

where $\mathrm{n}_{1}=\mathrm{u}-\mathrm{v}$ and $\mathrm{n}_{2}=\mathrm{w}-\mathrm{v}$.

Therefore, from Def. 2 and (3), if $X$ is H-convex, then we have

$$
\begin{aligned}
X & =\left(\bigcap_{\mathrm{H} \in \mathcal{R}(\mathcal{C o n v}(\mathrm{X}))} \mathrm{H}\right) \cap \mathbb{Z}^{3} \\
& =\bigcap_{\mathrm{H} \in \mathcal{R}(\mathcal{C} \operatorname{Conv}(\mathrm{X}))}\left(\mathrm{H} \cap \mathbb{Z}^{3}\right)
\end{aligned}
$$

where each $\mathrm{H} \cap \mathbb{Z}^{3}$ is called a digital half-space. It is plain that any digital half-space is H-convex.

In [13], [14], an efficient and simple algorithm was proposed for computing the convex hull of sets of 3D points, namely the Quickhull algorithm. It is based on a divide-and-conquer approach which is similar to the Quicksort. The algorithm has an average complexity $\mathcal{O}(n \log r)$ with $n$ the number of input points and $r$ is the number of output vertices (in the worst case, the algorithm has $\mathcal{O}\left(n^{2}\right)$ complexity). This algorithm is implemented not only for $X \subset \mathbb{Z}^{3}$ but more generally for $\mathrm{X} \subset \mathbb{R}^{3}$ in the CGAL library [15].

\section{B. Rigid motions of digital half-spaces}

The rigid motion of a digital half-space $H \cap \mathbb{Z}^{3}$ can be expressed as:

$$
\mathcal{T}_{\text {Conv }}\left(\mathrm{H} \cap \mathbb{Z}^{3}\right)=\mathfrak{T}(\mathrm{H}) \cap \mathbb{Z}^{3}
$$

where $\mathfrak{T}(\mathrm{H})$ is the rigid motion of $\mathrm{H}$ obtained analytically as follows.

It should be mentioned that digital objects and digital halfspaces involve exact computations with integers. In addition, as digital objects are finite in practice, we can assume hereafter only rational rigid motions, i.e. rotation matrices $R$ and translation vectors $t$ contain only rational elements.

The rational rigid motion $\mathfrak{T}$ is then applied on the integer half-space $\mathrm{H}$ defined by Eq. (4), to obtain a rational half-space:

$$
\mathfrak{T}(\mathrm{H})=\left\{x \in \mathbb{R}^{3} \mid \eta \cdot x+\delta \leq 0\right\}
$$

where $\eta=R \mathrm{n}$ and $\delta=\eta \cdot \mathrm{t}+d$.

Note that any rational half-space can be easily rewritten by an integer half-space in the form of Eq. (4).

\section{Rigid motions of H-convex objects}

In Sec. IV-B, we explained how to transform a digital halfspace using Eqs. (6) and (7). Since a $\mathrm{H}$-convex digital object $\mathrm{X}$ is represented by the intersection of a finite set of digital halfspaces generated from its convex hull, as shown in Eq. (5), we can define a rigid motion of $X$ on $\mathbb{Z}^{3}$ by using its associated digital half-spaces as:

$$
\begin{aligned}
\mathcal{T}_{\mathcal{C} \text { onv }}(\mathrm{X}) & =\mathfrak{T}\left(\bigcap_{\mathrm{H} \in \mathcal{R}(\mathcal{C} \text { onv }(\mathrm{X}))} \mathrm{H}\right) \cap \mathbb{Z}^{3} \\
& =\left(\bigcap_{\mathrm{H} \in \mathcal{R}(\operatorname{Conv}(\mathrm{X}))} \mathfrak{T}(\mathrm{H})\right) \cap \mathbb{Z}^{3} .
\end{aligned}
$$

The rigid motion $\mathcal{T}_{\mathcal{C} \text { onv }}(\mathrm{X})$ is called convexity preserving digital rigid motion. Roughly speaking, we transform each $\mathrm{H} \in \mathcal{R}(\operatorname{Conv}(\mathrm{X}))$ by $\mathfrak{T}$ and obtain the intersection of all $\mathfrak{T}(\mathrm{H})$ as the convex polyhedron $P$ such that $P=$ $\mathrm{H} \in \mathcal{R}(\operatorname{Conv}(\mathrm{X}))$

$\cap \mathfrak{T}(\mathrm{H})$. Then, we redigitize $P$ in order to obtain the transformed digital object $\mathcal{T}_{\mathcal{C} \text { onv }}(\mathrm{X})$.

Remark 1: The polygon corresponding to the convex hull of $\mathcal{T}_{\mathcal{C} \text { onv }}(\mathrm{X})$ is not equal, in general, to the transformed continuous polygon corresponding to the convex hull of $X$. However, we have the following inclusion relation

$$
\operatorname{Conv}\left(\mathcal{T}_{\text {Conv }}(\mathrm{X})\right) \subseteq \mathfrak{T}(\operatorname{Conv}(\mathrm{X}))
$$

and there is no grid point in $\mathfrak{T}(\operatorname{Conv}(\mathrm{X})) \backslash \operatorname{Conv}\left(\mathcal{T}_{\mathcal{C} \text { onv }}(\mathrm{X})\right)$. This leads the following property.

Property 1: If $\mathrm{X} \subset \mathbb{Z}^{3}$ is $\mathrm{H}$-convex, $\mathcal{T}_{\mathcal{C} \text { onv }}(\mathrm{X})$ is $\mathrm{H}$-convex.

As mentioned above, H-convexity does not imply connectivity in $\mathbb{Z}^{3}$ by contrast to $\mathbb{R}^{3}$. In the next section, we show that $\mathcal{T}_{\mathcal{C} \text { onv }}(\mathrm{X})$ remains not only $\mathrm{H}$-convex but also connected under a sufficient condition, called quasi-r-regularity, defined on the convex polyhedron $\operatorname{Conv}(\mathbf{X})$.

\section{Quasi-r-regularity and convexity preservation}

Let us now introduce the notion of quasi-r-regularity, that can be seen as an extension of $r$-regularity [4] in $\mathbb{R}^{3}$. Intuitively, a quasi- $r$-regular object $X \subset \mathbb{R}^{3}$ presents sufficient conditions for guaranteeing that its connectivity will not be affected by a Gauss digitization process.

Let us first introduce some notations and mathematical morphology notions. We note $B_{r}$ (resp. $S_{r}$ ) a close ball (resp. a sphere) of $\mathbb{R}^{3}$ of radius $r>0$. We note $\oplus, \ominus$ and $\circ$ the classical operators of dilation, erosion and opening commonly used in mathematical morphology [8], [16], [17]. In particular, $\oplus$ is the Minkowski addition, $\ominus$ the associated subtraction, and $\circ$ the composition of both, namely $X \circ Y=X \ominus Y \oplus Y$. Here, let us consider simple objects that are bounded, convex and simply connected (i.e., connected with no tunnel and no hole). Then, the $r$-regularity of Def. 1 on such a simple shaped object 


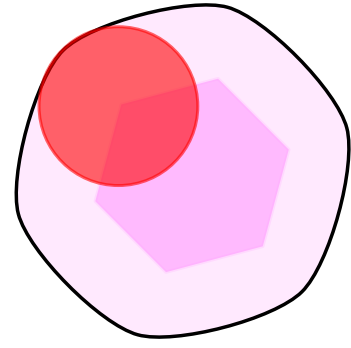

(a) $r$-regularity

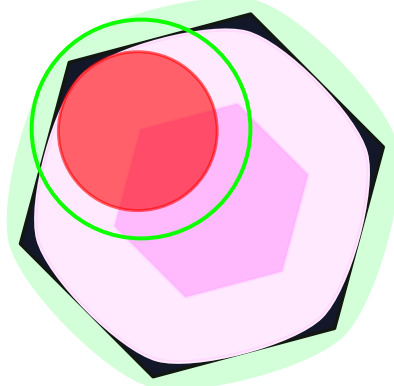

(b) Quasi-r-regularity
Fig. 2. Comparison between (a) $r$-regular [7] and (b) quasi- $r$-regular objects in 2D. While $r$-regular objects need to match perfectly its opening, quasi- $r$ regular objects are authorized to have a "margin" $r^{\prime}-r$ on the boundary and this allows non-smooth (for instance, non-differentiable, noisy...) boundary. The object $X \subset \mathbb{R}^{2}$ is in black, the ball $B_{r}$ and circle $S_{r^{\prime}}$ are respectively in red and green, the erosion $X \ominus B_{r}$ is in pink, the dilatation (resp. opening) $X \oplus B_{r}$ (resp. $X \circ B_{r}$ ) is in light pink and $X \ominus B_{r} \oplus C_{r^{\prime}}$ is in light green

$\mathrm{X} \subset \mathbb{R}^{3}$ can be written using those mathematical morphology notions as follows:

- $\mathrm{X} \ominus B_{r}$ is non-empty and connected,

- $\mathrm{X}=\mathrm{X} \ominus B_{r} \oplus B_{r}$.

Note that the convexity allows us to ignore the conditions on $\overline{\mathrm{X}}$. We now extend this $r$-regularity with some margin along the boundary of X.

Definition 3: Let $X \subset \mathbb{R}^{3}$ be a bounded, convex (and thus simply connected, i.e. connected with no tunnel and no hole) object. We say that $X$ is quasi-r-regular with margin $r^{\prime}-r$ (with $r^{\prime} \geq r>0$ ) if

- $X \ominus B_{r}$ is non-empty and connected; and

- $X \subseteq X \ominus B_{r} \oplus S_{r^{\prime}}$.

Remark 2: This definition does not require specific properties on the boundary of $X$. In particular, it need not be differentiable.

The difference between $r$-regular and quasi- $r$-regular objects is illustrated in Fig. 2 in two dimensions for simplicity. It is shown that $r$-regular objects need to match perfectly its opening, while quasi- $r$-regular objects are authorized to have a "margin" $r^{\prime}-r$ around the boundary and this allows nonsmooth boundary, such as polyhedral surfaces.

We are now ready to establish our main result on quasi$r$-regularity, that provides us with sufficient conditions for connectivity preservation.

Proposition 2: If $X$ is quasi-1-regular with margin $2 / \sqrt{3}-1$, then $\mathrm{X}=X \cap \mathbb{Z}^{3}$ and $\overline{\mathrm{X}}=\bar{X} \cap \mathbb{Z}^{3}$ are both connected.

Proof: We first prove the connectivity of $\mathrm{X}$. From Prop. 1, we have $\left(X \circ B_{1}\right) \cap \mathbb{Z}^{3}$ is connected since $1>\sqrt{3} / 2$. Let us consider the set $X \backslash\left(X \circ B_{1}\right)$. We now prove that any integer point $\mathrm{p} \in X \backslash\left(X \circ B_{1}\right)$ is adjacent to a point of $\left(X \circ B_{1}\right) \cap \mathbb{Z}^{3}$. We have $\mathrm{p} \in X \subseteq X \ominus B_{1} \oplus S_{2 / \sqrt{3}}$. Then, from the very definition of dilation, there exists $\mathrm{b} \in X \ominus B_{1}$ such that $\mathrm{b}$ is the center of a sphere $S_{2 / \sqrt{3}}$ (b) of radius $2 / \sqrt{3}$, and $\mathrm{p}$ is a point of this sphere. In particular, the distance between $b$ and $p$ is $2 / \sqrt{3}$. As $\mathrm{b}$ is a point of $X \ominus B_{1}$, it is also the center of a ball $B_{1}$ (b) of radius 1 included in $X \circ B_{1}$. From the definition of
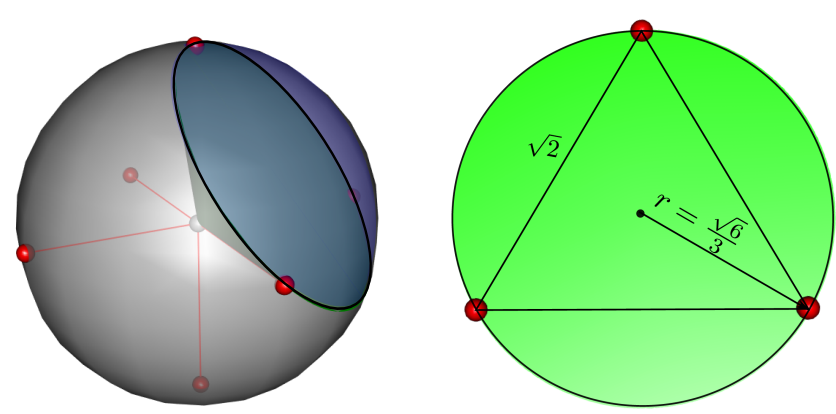

Fig. 3. Left: A spherical dome (in blue) lying on the sphere $S_{1}(\mathrm{p})$, whose boundary is a circle of radius $\sqrt{6} / 3$. Right: The circle forming this boundary encompasses an equilateral triangle of edge length $\sqrt{2}$ (in black). Then, the spherical dome always contains at least one (and at most 3) point(s) of $\mathbb{Z}^{3}$ adjacent to $\mathrm{p}$.

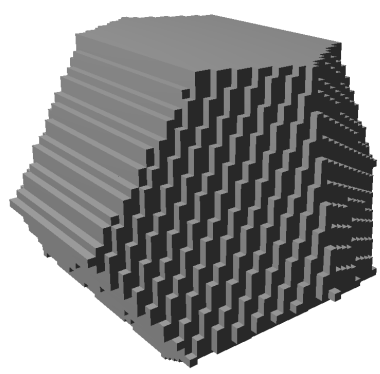

(a) $X$

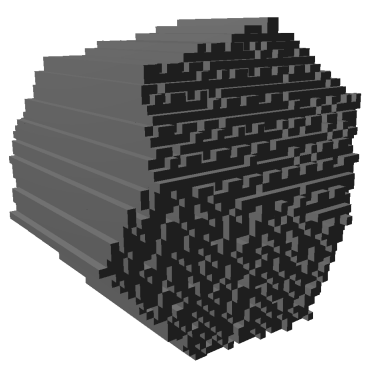

(b) $\mathcal{T}_{\text {point }}(\mathrm{X})$

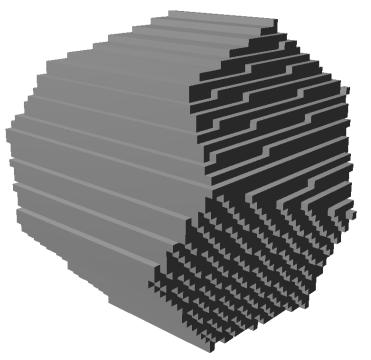

(c) $\mathcal{T}_{\mathcal{C} \text { onv }}(\mathrm{X})$
Fig. 4. A quasi-1-regular, H-convex, digital object $X$ (a) and its digital rigid motions by $\mathcal{T}_{\text {point }}(\mathrm{b})$ and (b) and $\mathcal{T}_{\mathcal{C} \text { onv }}(\mathrm{c})$.

adjacency, any point $q \in \mathcal{N}(p) \backslash\{p\}$ adjacent to $p$ belongs to the sphere $S_{1}(\mathrm{p})$ of radius 1 and center $\mathrm{p}$. Let us consider the intersection between $S_{1}(\mathrm{p})$ and $B_{1}(\mathrm{~b})$. It is a spherical dome, namely a part of the sphere $S_{1}(\mathrm{p})$ with a circular boundary. This circular boundary is the intersection of $S_{1}(\mathrm{p})$ and the spherical boundary of $B_{1}(\mathrm{~b})$. This intersection is a circle, that also corresponds to the intersection of $S_{1}(\mathrm{p})$ and the 2D plane orthogonal to the line (bp) and intersecting the segment $[\mathrm{bp}]$ at an equal distance of $1 / \sqrt{3}$ from both $b$ and $p$. Then, the radius of this circle is equal to $\sqrt{1^{2}-(1 / \sqrt{3})^{2}}=\sqrt{6} / 3$. Such circle exactly encompasses an equilateral triangle of edge length $\sqrt{2}$. As a consequence, the spherical dome of $S_{1}(\mathrm{p})$ bounded by this circle always contains at least one point $q \in \mathcal{N}(p) \backslash\{p\}$; see Fig. 3. As such point $q$ lies in $\left(X \circ B_{1}\right) \cap \mathbb{Z}^{3}$, it follows that $X$ is connected. The connectivity of $\bar{X}$ follows from the 


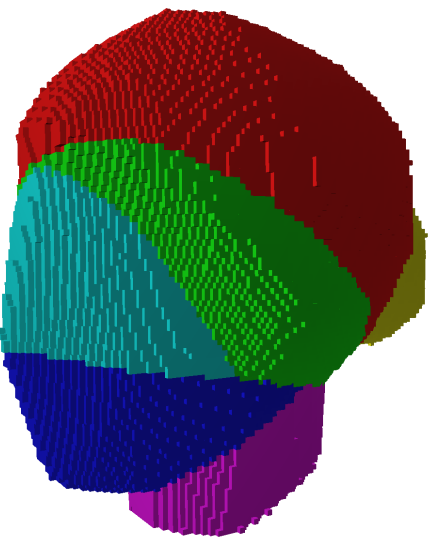

(a) $\mathrm{X}_{1}$

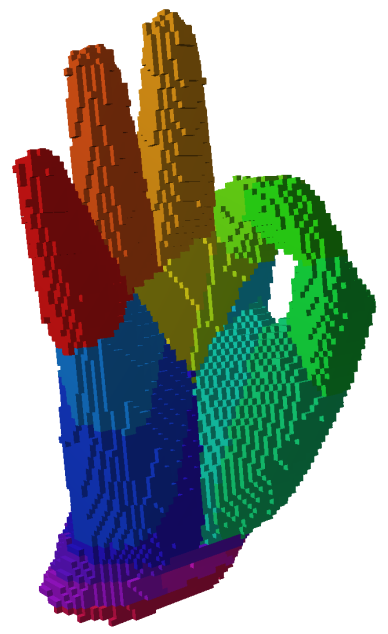

(d) $\mathrm{X}_{2}$

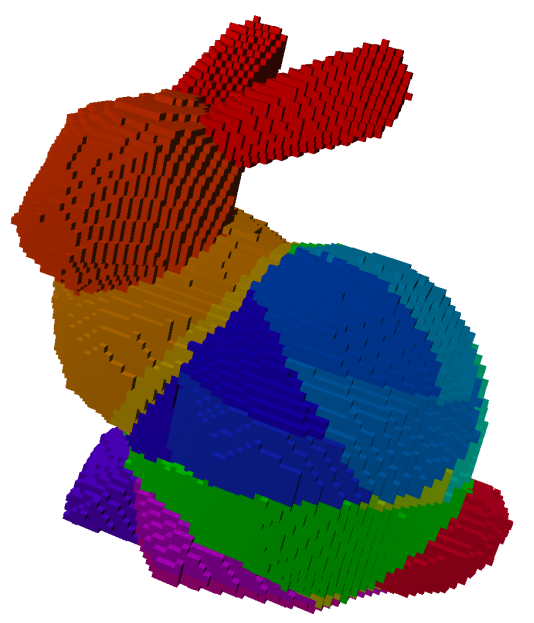

(g) $X_{3}$

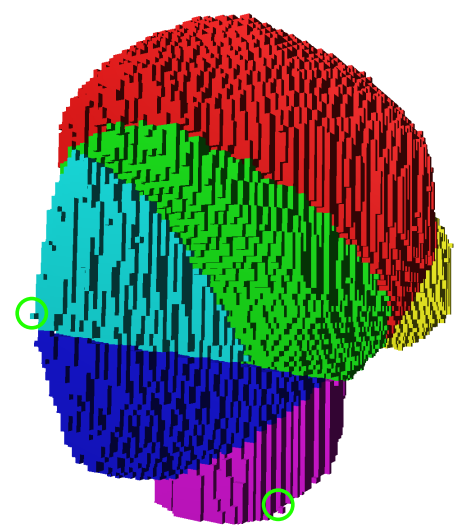

(b) $\mathcal{T}_{\text {point }}\left(\mathrm{X}_{1}\right)$

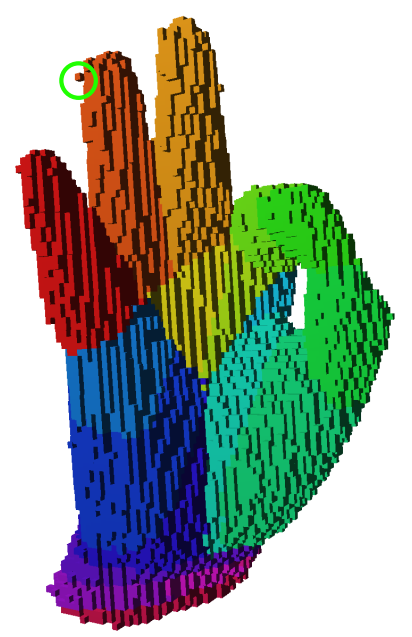

(e) $\mathcal{T}_{\text {point }}\left(\mathrm{X}_{2}\right)$

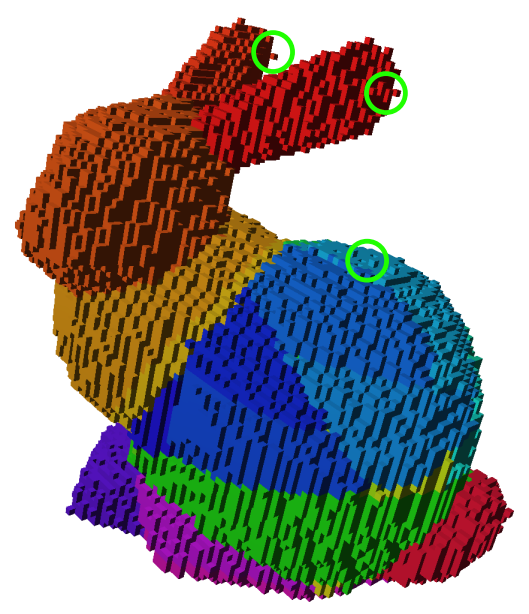

(h) $\mathcal{T}_{\text {point }}\left(\mathrm{X}_{3}\right)$

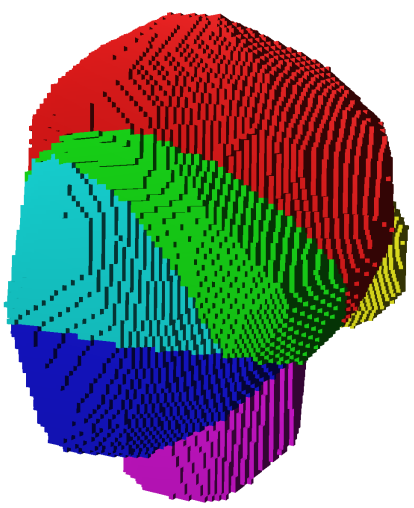

(c) $\mathcal{T}_{\text {Conv }}\left(\mathrm{X}_{1}\right)$

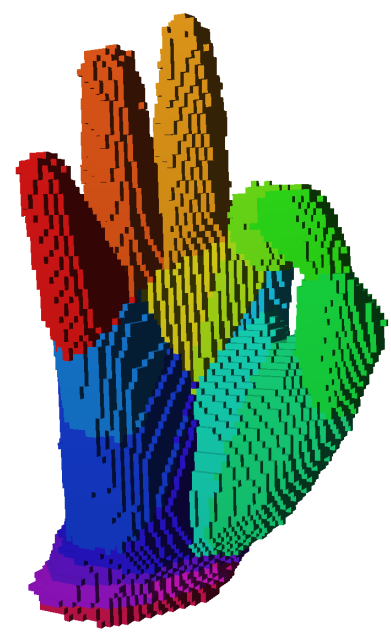

(f) $\mathcal{T}_{\mathcal{C} \text { onv }}\left(\mathrm{X}_{2}\right)$

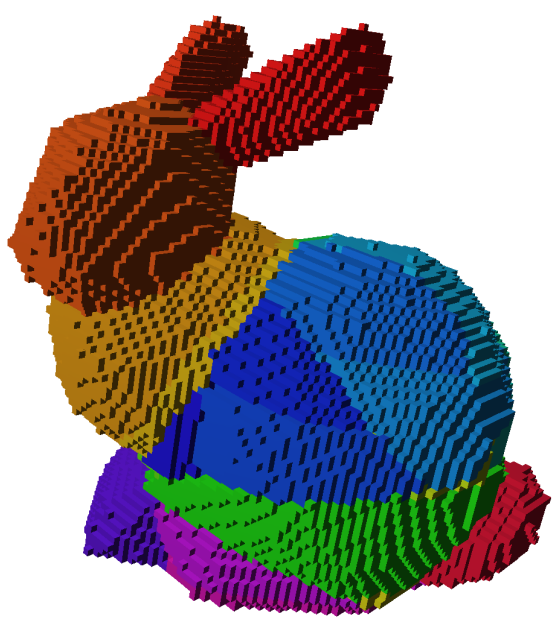

(i) $\mathcal{T}_{\text {Conv }}\left(\mathrm{X}_{3}\right)$

Fig. 5. Decomposition of non-convex objects into sets of H-convex, quasi-1-regular digital objects (a,d,g), and their rigid motions by $\mathcal{T}_{\text {point }}$ (b,e,h) and $\mathcal{T}_{\mathcal{C} \text { onv }}(\mathrm{c}, \mathrm{f}, \mathrm{i})$. Beyond the geometrical alterations, some examples of topological alterations of $\mathcal{T}_{\text {point }}$ are denoted by green circles. 
simple connectivity and convexity of $X$.

Remark 3: In particular, let us consider a convex polyhedron $P$ of $\mathbb{R}^{3}$. If $P$ is quasi-1-regular with margin $2 / \sqrt{3}-1$, then its digitization $P \cap \mathbb{Z}^{3}$ is connected.

In this article, we consider the family of $X$ being convex polyhedra, actually corresponding to the convex hulls of $\mathrm{H}$ convex digital objects. Then, we have the following result, that is a direct corollary of Property 1 and Proposition 2 .

Corollary 1: Let $\mathrm{X} \subset \mathbb{Z}^{3}$ be a $\mathrm{H}$-convex digital object. If $\operatorname{Conv}(\mathrm{X})$ is quasi-1-regular with margin $2 / \sqrt{3}-1$, then $\mathcal{T}_{\mathcal{C} \text { onv }}(\mathrm{X})$ is both H-convex and connected.

A digital object $\mathrm{X}$ that is $\mathrm{H}$-convex - i.e. such that $\mathrm{X}=$ $\operatorname{Conv}(\mathrm{X}) \cap \mathbb{Z}^{3}$ - is said to be quasi-1-regular if its convex hull $\mathcal{C}$ onv $(\mathrm{X})$ is quasi-1-regular with margin $2 / \sqrt{3}-1$. An example of quasi-1-regular digital object is given in Fig. 4(a).

The behaviour of $\mathcal{T}_{\mathcal{C} \text { onv }}$ compared to $\mathcal{T}_{\text {point }}$ when applied on a H-convex object is illustrated in Fig. 4. One can observe the better preservation of the shape of the transformed object, and in particular the preservation of $\mathrm{H}$-convexity by $\mathcal{T}_{\mathcal{C}}$ onv .

Figure 5 shows the result of $\mathcal{T}_{\mathcal{C} \text { onv }}$, compared to $\mathcal{T}_{\text {Point }}$, for non-convex digital objects via a convex decomposition process of non-convex objects [18]. Another approach can be used to handle non-convex digital objects, using the hierarchical object representation, e.g. the notion of concavity tree [19] in 3D.

\section{CONCLUSION}

A rigid motion method devoted to $3 \mathrm{D}$ digital convex objects has been proposed which is based on the half-space representation of a H-convex digital object. In order to guarantee the topological properties associated to $\mathrm{H}$-convexity preservation, we introduced the notion of quasi- $r$-regularity for convex continuous objects, including polyhedra. The proposed method works with digital objects satisfying the quasi-1-regular condition on their convex hull.

In practice, this property is not always true, thus limiting the use of our method. A multi-resolution approach would provide a promising way of tackling this issue. In addition, it is observed that quasi-1-regularity is sufficient but not always necessary for $\mathrm{H}$-convexity preservation. One of our perspectives is to find such sufficient and necessary condition. Study on non-convex digital objects will be more intensively investigated as well.

This work was partially funded by the French Agence Nationale de la Recherche, grant agreement ANR-15-CE400006 (CoMeDiC, https://lama.univ-savoie.fr/comedic) and by the French Programme d'Investissements d'Avenir (LabEx Bézout, ANR-10-LABX-58).

\section{REFERENCES}

[1] P. Ngo, Y. Kenmochi, N. Passat, and H. Talbot, "Topology-preserving conditions for 2D digital images under rigid transformations," Journal of Mathematical Imaging and Vision, vol. 49, no. 2, pp. 418-433, 2014

[2] P. Ngo, N. Passat, Y. Kenmochi, and H. Talbot, "Topology-preserving rigid transformation of $2 \mathrm{D}$ digital images," IEEE Transactions on Image Processing, vol. 23, no. 2, pp. 885-897, 2014.

[3] P. Ngo, Y. Kenmochi, I. Debled-Rennesson, and N. Passat, "Convexitypreserving rigid motions of 2D digital objects," in Proceedings of DGCI ser. LNCS, vol. 10502, 2017, pp. 69-81.
[4] R. Klette and A. Rosenfeld, Digital geometry: Geometric Methods for Digital Picture Analysis. Amsterdam, Boston: Elsevier, 2004.

[5] P. Stelldinger and U. Köthe, "Towards a general sampling theory for shape preservation," Image and Vision Computing, vol. 23, no. 2, pp. 237-248, 2005.

[6] P. Stelldinger, L. J. Latecki, and M. Siqueira, "Topological equivalence between a 3D object and the reconstruction of its digital image," IEEE Transactions on Pattern Analysis and Machine Intelligence, vol. 29, no. 1, pp. 126-140, 2007.

[7] T. Pavlidis, Algorithms for Graphics and Image Processing. Berlin: Springer, and Rockville: Computer Science Press, 1982.

[8] J. Serra, Image Analysis and Mathematical Morphology. Orlando, FL, USA: Academic Press, Inc., 1983.

[9] C. E. Kim, "On the cellular convexity of complexes," IEEE Transactions on Pattern Analysis and Machine Intelligence, vol. 3, no. 6, pp. 617-625, 1981.

[10] U. Eckhardt, "Digital lines and digital convexity," in Digital and Image Geometry: Advanced Lectures, G. Bertrand, A. Imiya, and R. Klette, Eds. Springer Berlin Heidelberg, 2001, pp. 209-228.

[11] G. Cristescu and L. Lupsa, Non-Connected Convexities and Applications. Dordrecht: Kluwer Academic Publishers, 2002.

[12] P. McMullen and G. C. Shephard, Convex Polytopes and the Upper Bound Conjecture. Cambridge University Press, 1971.

[13] C. B. Barber, D. P. Dobkin, and H. Huhdanpaa, "The quickhull algorithm for convex hulls," ACM Transactions on Mathematical Software, vol. 22, no. 4, pp. 469-483, 1996.

[14] K. Mehlhorn, S. Näher, T. Schilz, S. Schirra, M. Seel, R. Seidel, and C. Uhrig, "Checking geometric programs or verification of geometric structures," in Proceedings of SCG, 1996, pp. 159-165.

[15] "CGAL: The computational geometry algorithms library," http://www. cgal.org.

[16] H. Heijmans and C. Ronse, "The algebraic basis of mathematical morphology. I Dilations and erosions," Computer Vision, Graphics, and Image Processing, vol. 50, no. 3, pp. 245-295, 1990.

[17] C. Ronse and H. Heijmans, "The algebraic basis of mathematical morphology: II. Openings and closings," CVGIP: Image Understanding, vol. 54, no. 1, pp. 74-97, 1991.

[18] K. Mamou and F. Ghorbel, "A simple and efficient approach for 3D mesh approximate convex decomposition," in Proceedings of ICIP, 2009, pp. 3465-3468.

[19] J. Sklansky, "Measuring concavity on a rectangular mosaic," IEEE Transactions on Computers, vol. C-21, no. 12, pp. 1355-1364, 1972. 